\title{
Genetic Bleeding Risk Score (GBRS) for Patients on Oral Anticoagulant Therapy
}

Risha Nahar ${ }^{1,2 *}$, Ishwar C Verma1, Roumi Deb ${ }^{2}$, Renu Saxena ${ }^{1}$, Parul Takkar $^{3}$, Sujay Shad ${ }^{4}$, Rajiv Parakh ${ }^{5}$ and Prahlad K Sethi ${ }^{6}$

${ }^{1}$ Center of Medical Genetics, Sir Ganga Ram Hospital, New Delhi, India

${ }^{2}$ Amity Institute of Biotechnology, Amity University, Noida, Uttar Pradesh, India

${ }^{3}$ Department of Research, Sir Ganga Ram Hospital, New Delhi, India

${ }^{4}$ Department of Cardiac Surgery, Sir Ganga Ram Hospital, New Delhi, India

${ }^{5}$ Division of Peripheral Vascular \& Endovascular sciences, Medicity Medanta Hospital, New Delhi, India

${ }^{6}$ Department of Neurology, Sir Ganga Ram Hospital, New Delhi, India

\begin{abstract}
Aims: The present study focussed on deriving and validating a 'genetic bleeding risk score' (GBRS) based on genetic and non-genetic factors associated with bleeding in patients on long term anticoagulation therapy.

Patients and Methods: Patients on warfarin $(n=53)$ or acenocoumarol $(n=257)$ long-term therapy were genotyped for twenty one SNPs in six genes. Two GBRSs were developed and validated.

Results: The incidence rate was 16.86 and 4.46 per 100 person-years for minor and major bleeding respectively. The novel GBRS (positive predictive value $=83.3 \%$, specificity $=97.4 \%$ ) comprised of four parameters; age $>65$ years, F5 rs6025, VKORC1 rs9934438 and CYP2C9 rs 1057911.
\end{abstract}

Conclusions: The present study is the first to devise and validate a genetic based score for predicting bleeding among first time users of oral anticoagulants.

Keywords: Bleeding; Oral anticoagulants; Hemorrhage; Adverse drug reactions; Genetic bleeding risk; CYP2C9; VKORC1; Risk score; Acenocoumarol; Warfarin

\section{Introduction}

Natural dicoumarol was first identified in year 1940 in mouldy hay as a cause of serious hemorrhagic diathesis in cattle [1]. Further research led to the development of warfarin, a coumarin derivative that was initially promoted as a rat poison and first showed success in prophylaxis of deep vein thrombosis in year 1941 [2]. It is now the most widely used anticoagulant in the treatment and prevention of thrombosis. Despite its common usage, oral anticoagulant (OAC) therapy is associated with significant bleeding complications. Several hospital based studies in India [3,4] and the world [5,6] have ranked anticoagulant-induced bleeding as the most common cause amongst $5 \%-6.9 \%$ of hospital admissions that occur due to adverse drug reactions.

Both, genetic (CYP2C9 and VKORC1 variants) [7-12] and non genetic factors (drug-drug interactions, additional medical conditions, age, history of bleeding) $[13,14]$ are known to contribute towards bleeding or hemorrhage in patients on oral anticoagulant therapy. Several pharmacogenetic dosing algorithms for warfarin [15-18] and acenocoumarol [19-21] have been developed so far. A systematic review and meta analysis [22] aimed to investigate the efficacy of genotype-guided dosing of warfarin in reducing bleeding events and over-anticoagulation included three randomised clinical trials [23-25] that compared pharmacogenetic dosing with a standard dose control algorithm in patients starting warfarin for the first time. None of the above studies showed a statistically significant difference in bleeding rates between the two groups. This is possibly because these studies attempted to predict bleeding using a pharmacogenetic 'dosing' algorithm originally derived by analyzing dosage in patients, rather than a true bleeding prediction algorithm that, on the other hand should ideally be derived by analyzing bleeding outcomes in patients.
Hence, it would be unreasonable to discount the role of genetic variants in predicting the risk of bleeding.

Although, a few bleeding risk prediction scores are available $[13,14,26-28]$, most are derived from the white population and more importantly, none have evaluated the predictive significance of genetic risk factors so far. The HEMOR 2 RHAGES score formulated by previously recognized bleeding risk factors from the literature includes CYP2C9 variants as one of the variables in their score index; however its predictive usefulness in the cohort was neither evaluated nor validated due to non availability of DNA [28]. The present study focussed on deriving and validating a 'Genetic bleeding risk' (GBR) score based on genetic and non-genetic factors associated with bleeding (both minor and major) in patients on long term anticoagulation therapy. Apart from variants in CYP2C9 and VKORC1 genes, variants in $A P O E$, $A B C B 1$ (MDR1), CYP4F2, F5 and F2 were also analysed in the current study.

Coumarin derivatives interfere with the recycling of vitamin $\mathrm{K}$ in the liver. Vitamin $\mathrm{K}$ is involved in the carboxylation of the precursor proteins for the coagulation factors II, VII, IX and X. In the presence of coumarin derivative, the activity of these components is lowered thereby inhibiting coagulation. The transport of vitamin $\mathrm{K}$ to the liver is dependent on apolipoprotein $\mathrm{E}(A P O E)$. The prevalence of

*Corresponding author: Risha Nahar, PhD Scholar, Center of Medical Genetics, Sir Ganga Ram Hospital, Rajinder Nagar, New Delhi-110060, India, Tel: +919849507575; Fax: 011-25862206; E-mail: rishanahar@gmail.com

Received May 06, 2013; Accepted June 24, 2013; Published June 26, 2013

Citation: Nahar R, Verma IC, Deb R, Saxena R, Takkar P, et al. (2013) Genetic Bleeding Risk Score (GBRS) for Patients on Oral Anticoagulant Therapy. Int $J$ Genomic Med 1: 101. doi:10.4172/2332-0672.1000101

Copyright: () 2013 Nahar R, et al. This is an open-access article distributed under the terms of the Creative Commons Attribution License, which permits unrestricted use, distribution, and reproduction in any medium, provided the original author and source are credited. 
APOE isoforms (e2, e3, e4; distinguished by two non synonymous polymorphisms; rs7212 and rs229358) varies by race, and each isoform has varying ability to facilitate clearance of vitamin-K-rich lipoproteins from plasma [29]. The e4 allele has been associated with higher warfarin dose among African Americans and Italians, but not Caucasians $[30,31]$. However, none of the studies have analysed the association of $A P O E$ isoforms with anticoagulant-induced bleeding.

The intestinal bioavailability of oral anticoagulant drugs and their transport in cellular systems is dependent on the efflux pump P-glycoprotein, encoded by the adenosine triphosphate-binding cassette $(A B C B 1)$ gene (multidrug resistance gene, MDR1) [32,33]. The synonymous $3435 \mathrm{~T}$ variant (closely linked to $2677 \mathrm{~T}>\mathrm{G}$ and $1236 \mathrm{~T}>\mathrm{C}$ ) has been frequently observed among patients requiring low dose of warfarin [34]. Another study [35] reported that $A B C B 1$ $2677 \mathrm{GG} / 3435 \mathrm{CC}$ haplotype was associated with lower dose, while the $2677 \mathrm{TT} / 3435 \mathrm{TT}$ and $2677 \mathrm{GT} / 3435 \mathrm{TT}$ haplotypes were associated with higher dose of acenocoumarol. These reports suggest that an assessment of these variants could be useful for predicting P-gp-dependent adverse drug reactions with oral anticoagulants.

Genome wide association studies have recognized CYP4F2 rs2108622 (Val433Met) as a significant contributor of inter individual variation in coumarin dose requirement, however its effect size is smaller than that of CYP2C9 and VKORC1 variants [36,37]. Protein CYP4F2 catalyzes many reactions involved in drug metabolism. It is hypothesized that CYP4F2 might interfere in the vitamin $\mathrm{K}$ recycling or could be involved in the metabolism of acenocoumarol as the rs 210862 polymorphism is associated with varied levels of FII, FVII, FIX, and FX after acenocoumarol therapy [37]. There are no studies until now that have analyzed the rs 210862 variant in correlation with anticoagulantinduced bleeding.

The most common inherited predispositions to thrombophilia are Factor V Leiden (FVL, coagulation factor V) and the prothrombin (coagulation factor II) rs1799963 mutation which result in activated protein $\mathrm{C}$ resistance or elevated concentrations of prothrombin (the immediate precursor of thrombin) respectively [38]. Factor V Leiden mutation (rs6025) was observed to play a prohemorrhagic role among patients on anticoagulant therapy in a study by Castori et al. [39]. There is a dearth of further studies analysing the association of FVL in bleeding with oral anticoagulants.

\section{Patients and Methods}

\section{Setting and outcomes}

The study was conducted at the Sir Ganga Ram Hospital, a tertiary health care centre in New Delhi, India. The research protocol was approved by the Ethics Board Committee of Sir Ganga Ram Hospital and is in accordance with the ethical standards of Declaration of Helsinki (World Medical Association). Patients were enrolled from the outpatient clinic of Department of vascular surgery and inpatients from the Department of Cardiac surgery. All participants gave written informed consent. Primary outcome of the present study was drug-induced bleeding. The 'case' definition in the present study was the patient who develops bleeding during oral anticoagulation therapy and with oral anticoagulants along with other drugs (drugdrug interaction). Choice of the anticoagulant for the patients and management of anticoagulation therapy was carried out by the respective clinicians. All patients were started on a standard dosing scheme of acenocoumarol (ACITROM, $2 \mathrm{mg}$ per day from days 1 to 3) or warfarin (WARF, $5 \mathrm{mg}$ per day from days 1 to 4 ) with a target
INR range of 2-3. Doses were adjusted on the basis of the INR of the patient thereafter. Prothrombin times and International normalized ratios (INRs) were evaluated once every 1 to 4 weeks depending on the stability of the INR and anticoagulation level. All study participants were followed up for approximately 15 months from the time of their first initiation dose until the end of study period or withdrawal of oral anticoagulation therapy.

\section{Cohort}

Patients $\geq 18$ years of age, initiated (first time) on oral anticoagulant therapy and anticipated long term ( $>2$ years) treatment duration were eligible. Since the aim of the study was to recognize novel genetic and non-genetic bleeding predictors, patients with known obvious risk factors such as abnormal kidney function (e.g., chronic dialysis, renal transplantation), abnormal liver function (eg, liver cirrhosis), history of bleeding and malignancy were excluded. Of 1483 patients visiting the clinic during the study period, only 310 (20.9\%) patients fit the eligibility criteria and were enrolled in the study. The rest of the patients either lacked a clinical indication for long-term oral anticoagulation therapy (437, 29.5\%), or were previously on anticoagulation therapy (680, $45.9 \%)$, or were less than 18 years of age $(4,0.27 \%)$, or had comorbid conditions such as cancer, renal disease or history of bleeding $(52,3.5 \%)$. Of those on acenocoumarol therapy $(n=257), 20 \%$ of patients $(n=51)$ were randomly selected to form the validation cohort (Cohort ${ }^{\mathrm{Val}}$ ), while the rest served as the derivation cohort for acenocoumarol (Cohort ${ }^{\mathrm{AC}}$; $\mathrm{n}=206$ ) and warfarin (Cohort ${ }^{\mathrm{WF}} ; \mathrm{n}=53$ ). Warfarin was less commonly used; hence validation cohort for the same was not available. Four ml of peripheral blood was collected from the participating patients during their first clinic visit. For the period of follow-up the patients were regularly assessed for their INR values, change in dosage, concomitant use of drugs and bleeding complications. Since it is known that variable dietary intake of vitamin $\mathrm{K}$ can have profound effects on the risk of bleeding, patients had been therefore counselled regarding the same. They were advised to maintain a stable intake of vitamin K-containing foods in their diet. Patient compliance to diet and adherence to therapy was checked during every follow up.

\section{Assessment of bleeding}

Bleeding complications were initially classified as minor (requiring no additional testing, referral, or outpatient visits), or major (requiring medical or surgical intervention, major blood loss requiring blood transfusion of two units or more). For the purpose of developing a bleeding prediction score, both minor and major bleeding episodes were pooled to enable the forecast of any type of bleeding among OAC users. The rationale for this is that although majority of bleeding is clinically mild, patients with minor bleeds have a significantly increased relative risk (2.9) of subsequent major bleeding as compared to those without any minor bleeding [40,41]. Hence, detection of minor bleeding in addition to major bleeding is clinically crucial as well. Univariate analyses were performed separately in patients on acenocoumarol $\left(\right.$ Cohort $\left.^{\mathrm{AC}}\right)$ and warfarin $\left(\right.$ Cohort $\left.^{\mathrm{WF}}\right)$ in addition to the pooled derivation cohort on both types of anticoagulants (Cohort ${ }^{\mathrm{AC}+\mathrm{WF}}$ ).

\section{Selection of candidate SNPs}

Twenty one SNPs in seven different genes were selected for analysis. The method of selection of SNPs in CYP2C9 and VKORC1 is detailed in supplementary material (Supplemental material 1).

\section{Genotyping methods}

CYP2C9, VKORC1, CYP4F2: In addition to the three common 
variants associated with coumarin response: CYP2C ${ }^{\star} 2$ (rs1799853/ $430 \mathrm{C}>\mathrm{T} / \mathrm{p} . \mathrm{Cys} 144 \mathrm{Arg}$ in exon 3$), \mathrm{CYP} 2 \mathrm{C}^{*} 3(\mathrm{rs} 1057910 / \mathrm{c} .1075 \mathrm{~A}>\mathrm{C} /$ p.Ileu359Leu in exon 7) and VKORC1-1639G $>$ A (rs9923231/ g.3588G $>\mathrm{A}$ in upstream promoter region), the above mentioned variants were genotyped by resequencing in the remaining patients as well. CYP4F2 rs2108622 (c.1297G>A/ p.Val433Met in exon 11) was also genotyped. All of the above SNPs in the CYP2C9, VKORC1 and CYP4F2 genes were analyzed by means of allele-specific PCR or amplification refractory mutation system (ARMS) PCR using special primers designed with BatchPrimer3 [42-44] (primers available on request).

MDR1/ABCB1: The three common polymorphisms in the MDR1/ $A B C B 1$ gene; rs1128503 (c.1236T $>C /$ p.Gly412Gly in exon 12), rs2032582 (2677T>G/A/ Ser893Ala/Thr in exon 21) and rs1045642 (3435C $>\mathrm{T} /$ Ile1145Ile in exon 26$)$ that are implicated in variable drug response were genotyped using previously published methods $[45,46]$.

APOE: The APOE isoforms (e2, e3, e4) that are distinguished by two non synonymous polymorphisms; rs7212 and rs229358 were detected using previously published primer sequences [47] for PCR followed by restriction enzyme HhaI cleavage of the amplified product to generate allele discriminating DNA fragments.

F5 and F2: Genotyping of factor V Leiden variant (rs6025/ $1691 \mathrm{G}>\mathrm{A}$ ) and prothrombin mutation (rs1799963/20210G $>$ A) was carried out by restriction enzyme digestion of PCR-amplified DNA based on previously published protocols $[48,49]$ with modifications.

Appropriate quality control was carried out with wild type and variant genotype control samples. Internal controls were used with each allele specific primer pair to check for DNA and PCR reaction quality. The first 20 samples genotyped for each of the above SNPs were confirmed by resequencing results additionally all genotypes were confirmed by repeating the test. PCR was carried by standard protocol using 10 pico moles of each primer.

\section{Statistical analysis}

All data analysis was performed with SPSS, version 16.0 (SPSS Inc., Chicago, IL). Assuming a bleeding incidence of $10 \%$ in individuals on oral anticoagulants and considering an absolute difference of $10 \%$ (from 5 to 15\%) as being worth detecting the sample size needed to be at least 150 patients, with an $\alpha$ error of 0.05 and a statistical power of 0.9 . Two by two contingency chi-squares were used to compare allele frequencies between groups. The expected genotype frequencies and the deviation from Hardy-Weinberg equilibrium were analyzed by Chi square test. The presence of any differences between the groups with (cases) and without bleeding (controls) was tested by Fisher exact test for categorical variables and by independent samples $t$ test for continuous variables. All potential bleeding risk factors identified from the univariate analyses with a $p$ value $<0.05$, were included in the multivariate logistic regression analyses. All comparisons were twotailed. Variables with $\mathrm{p}<0.05$ in the final model were considered to be significant contributors and were checked for interaction effects. Two independent genetic bleeding risk scores (GBRS) were designed to predict risk of bleeding based on significant factors from Cohort ${ }^{\mathrm{AC}}$ and pooled Cohort ${ }^{\mathrm{AC}+\mathrm{WF}}$. Based on their respective multivariate regression coefficients (Beta), scores were allotted for each of the bleeding risk factors in the final model. Scores were awarded to all patients (in derivation cohort) accordingly. Bleeding risk scores were then stratified into low and high risk. To measure the discriminative power of the scoring systems, receiver operating characteristic (ROC) graphs were plotted by taking the predictive probabilities as the test variable. The c-statistic (area under the curve, AUC) that reflects the concordance of predicted and observed bleeding episodes was evaluated for both regression models among different patient subgroups. An AUC < 0.5 meant lack of discriminative power and $\mathrm{AUC}=1.0$ meant perfect discriminative capacity of the score system.

\section{Validation of GBRS}

The GBRS was validated in an independent patient cohort on acenocoumarol therapy $\left(n=51\right.$; Cohort $\left.{ }^{\mathrm{Val}}\right)$. Specific scores were awarded for the presence of risk factors from the model and added up based on which the patients were designated into the two risk categories; low and high. A ROC was plotted and the AUC (C statistic) was calculated to measure the predictive accuracy of the two models derived from Cohort ${ }^{\mathrm{AC}}$ and pooled Cohort ${ }^{\mathrm{AC}+W F}$. The sensitivity and specificity of GBRS to predict bleeding accurately was compared with that of a clinical bleeding risk score (CBRS) score. The CBRS was derived in a similar method as the GBRS, excluding the genetic variables. In addition, we report the AUC statistics in a subgroup analysis of individuals with OAC combined with an antiplatelet drug, with venous thromboembolism and deep vein thrombosis (DVT) as clinical indication for OAC.

\section{Results}

\section{Characteristics of patient population}

The study population had a mean age of 42.51 years (standard deviation, $\mathrm{SD}=17.36)$ and an average $\mathrm{BMI}$ of $25.82(\mathrm{SD}=5.8)$. The mean follow up period was 475.32 days $(S D=172.57)$ during which an average of $17.04(\mathrm{SD}=5.31)$ INR measurements were recorded for each patient. The study population had $32.98 \%(\mathrm{SD}=18)$ of INRs within the therapeutic range, $13.28 \%(\mathrm{SD}=15.61)$ INRs $>3.0$ and $53.73 \%$ ( $S D=20.89)$ INRs $<2.0$. Among the 294 patients who stabilized on either anticoagulant, the average time taken to stabilize was 82.9 days $(\mathrm{SD}=65.31)$ and the mean stabilized weekly dose was $20.03 \mathrm{mg}$ $(\mathrm{SD}=8.21)$ and $43.01 \mathrm{mg}(\mathrm{SD}=16.34)$ of acenocoumarol and warfarin respectively.

The demographic, clinical characteristics and clinical parameters of anticoagulation therapy in the two derivation cohorts (on warfarin and acenocoumarol) and validation cohort (on acenocoumarol) are detailed in Supplement Table 1. No significant difference was observed either between Cohort ${ }^{\mathrm{AC}}$ and Cohort ${ }^{\mathrm{Val}}$ or Cohort ${ }^{\mathrm{WF}}$ and Cohort ${ }^{\mathrm{Val}}$. Some characteristics such as gender, clinical indications, certain concomitant drugs and follow up time were observed to differ in the patient groups on acenocoumarol $\left(\right.$ Cohort $\left.^{\mathrm{AC}}\right)$ and warfarin (Cohort $\left.{ }^{\mathrm{WF}}\right)$. The genotype and allele frequency of all SNPs are tabulated (Supplementary Table 2) and found to fit in Hardy Weinberg equilibrium. The rate of bleeding in patients was consistent with both anticoagulants $(\mathrm{p}=0.532)$. About onethird ( $\mathrm{n}=26,30.23 \%)$ of bleeding complications occurred in patients with low INRs $(<3.0)$. During the study period, $68(21.9 \%)$ patients presented with minor bleeding (mild nasal bleeds, mild bruising or minor oral bleeds) and $18(5.8 \%)$ with major bleeding (major dermatologic bleeds, gastrointestinal bleeds or genitourinary bleeds). The incidence rate was $21.32,16.86$ and 4.46 per 100 person-years for any type of bleeding, minor bleeding and major bleeding respectively.

\section{Quality of anticoagulation in bleeders and non-bleeders}

Variations with respect to quality of anticoagulation therapy among the cases and controls in each cohort were analyzed. A linear positive relationship with bleeding was observed with over anticoagulation 
Citation: Nahar R, Verma IC, Deb R, Saxena R, Takkar P, et al. (2013) Genetic Bleeding Risk Score (GBRS) for Patients on Oral Anticoagulant Therapy. Int J Genomic Med 1: 101. doi:10.4172/2332-0672.1000101

(INR $>6$ ) and per cent of INRs $\geq 3$ and a negative linear association of bleeding with per cent of INRs $\leq 2$ was found to be significant in both warfarin and acenocoumarol, and the pooled cohort (Supplementary Table 3). Patients with INR $>3.0$ were observed to be more likely to bleed with acenocoumarol. Bleeders on warfarin took a longer time to stabilize than the non-bleeders. The stabilized weekly acenocoumarol dose was observed to be lower for bleeders as compared to non- bleeders. Higher numbers of INR measurements were recorded from bleeders than non-bleeders in the warfarin and pooled cohort.

\section{Derivation and evaluation of bleeding risk indices}

Univariate analyses for bleeding complications revealed six bleeding predictors in the warfarin cohort, five in acenocoumarol cohort and nine predictors in the pooled cohort (Table 1). The final best fitting models derived by multivariate regression in cohort $^{\mathrm{AC}}\left(\mathrm{GBRS}^{\mathrm{AC}}\right)$

\begin{tabular}{|c|c|c|c|c|c|c|c|c|c|}
\hline \multirow{2}{*}{$\begin{array}{c}\text { Variables }^{* *} \\
\% *\end{array}$} & \multicolumn{3}{|c|}{ Warfarin $(n=53)$} & \multicolumn{3}{|c|}{ Acenocoumarol $(n=206)$} & \multicolumn{3}{|c|}{ Pooled ( $n=259)$} \\
\hline & Case $\mathrm{N}=18$ & Control $\mathrm{N}=35$ & $P$ value & Case $\mathrm{N}=55$ & Control $\mathrm{N}=151$ & $P$ value & Case $\mathrm{N}=73$ & Control $\mathrm{N}=186$ & $P$ value \\
\hline CYP2C9 rs1057911 & 55.6 & 5.7 & $<0.001$ & 25.5 & 16.6 & 0.149 & 32.9 & 14.5 & 0.001 \\
\hline CYP2C9 rs9332230 & 50.0 & 11.4 & 0.005 & 32.7 & 25.8 & 0.327 & 37 & 23.1 & 0.024 \\
\hline CYP2C9 rs9332172 & 50.0 & 22.9 & 0.045 & 43.6 & 32.5 & 0.138 & 45.2 & 30.6 & 0.027 \\
\hline CYP2C9 rs1057910\$ & 38.9 & 17.1 & 0.081 & 36.4 & 23.2 & 0.058 & 37 & 22 & 0.014 \\
\hline CYP2C9 rs2298037 & 61.1 & 45.7 & 0.288 & 41.8 & 55.6 & 0.079 & 46.6 & 53.8 & 0.298 \\
\hline VKORC1 rs7294 & 44.4 & 45.7 & 0.930 & 58.2 & 44.4 & 0.079 & 54.8 & 44.6 & 0.14 \\
\hline VKORC1 rs55894764 & 0.0 & 2.9 & 0.469 & 5.5 & 0.7 & 0.027 & 7.7 & 0 & 0.255 \\
\hline VKORC1 rs9934438 & 22.2 & 14.3 & 0.469 & 47.3 & 28.5 & 0.011 & 41.1 & 25.8 & 0.016 \\
\hline ABCB1 rs2032582 & 50.0 & 62.9 & 0.368 & 49.1 & 64.9 & 0.04 & 49.3 & 64.5 & 0.025 \\
\hline F5 rs6025 & 38.9 & 5.7 & 0.005 & 10.9 & 7.3 & 0.403 & 17.8 & 7 & 0.009 \\
\hline Proton pump inhibitor & 11.1 & 14.3 & 1.0 & 29.1 & 15.9 & 0.034 & 24.7 & 15.6 & 0.089 \\
\hline Antiplatelet & 77.8 & 28.6 & 0.001 & 70.9 & 62.3 & 0.250 & 72.6 & 55.9 & 0.013 \\
\hline Statin $\$$ & 0.0 & 28.6 & 0.011 & 34.5 & 21.9 & 0.064 & 30.8 & 15.8 & 0.253 \\
\hline Arterial occlusive disease & 0.0 & 5.7 & 0.543 & 16.4 & 7.9 & 0.077 & 12.3 & 7.5 & 0.222 \\
\hline Age >65 years; mean & 16.7 & 2.9 & 0.108 & 27.3 & 7.3 & $<0.001$ & 24.7 & 6.5 & $<0.001$ \\
\hline
\end{tabular}

* Unless indicated otherwise

** Only the variables that showed significant correlation in either one or more cohorts are included in this table.

'Cases' represent the patients who experienced one or more bleeding episodes while 'controls' refer to patients who did not bleed during the long term follow up.

$\$$ Protective effect, negative correlation with bleeding. All factors that showed a significance level of $p<0.10$ are tabulated above, although only the ones with $p<0.05$ (in bold font) were included in the logistic multivariate regression model.

$\$ \$ A n$ independent correlation analysis of CYP2C9 rs1799853 and rs1057910 variants among the patients with major bleeding events ( $n=18$ ) revealed a significant association of rs 1057910 variant allele with major bleeding $(p=0.001)$

Table 1: Bleeding predictors in univariate analysis

\begin{tabular}{|c|c|c|c|c|c|c|}
\hline \multirow{2}{*}{$\begin{array}{l}\text { Bleeding predictor } \\
\text { variables }\end{array}$} & \multicolumn{2}{|c|}{ GBRS $^{A C}$} & \multicolumn{2}{|c|}{ GBRS $^{A C+W F}$} & \multicolumn{2}{|c|}{$\mathrm{CBRS}^{\mathrm{AC}+\mathrm{WF}}$} \\
\hline & B & Exp B $(95 \% \mathrm{Cl})$ & B & Exp B $(95 \% \mathrm{Cl})$ & B & $\operatorname{Exp~B~}(95 \% \mathrm{Cl})$ \\
\hline Factor V Leiden & GBRSAC+WF & & 1.12 & $3.08(1.26-7.51)$ & & \\
\hline Age $>65$ years & 1.42 & $4.14(1.71-10.06)$ & 1.60 & $4.95(2.13-11.48)$ & 1.5 & $4.46(2.0-9.93)$ \\
\hline VKORC1 rs9934438 & 0.82 & $2.27(1.28-4.03)$ & 0.74 & $2.09(1.25-3.51)$ & & \\
\hline VKORC1 rs55894764 & 2.47 & $11.86(1.13-124.77)$ & & & & \\
\hline CYP2C9 rs1057911 & & & 1.13 & $3.08(1.56-6.1)$ & & \\
\hline Antiplatelet & & & & & 0.67 & $1.95(1.06-3.57)$ \\
\hline
\end{tabular}

GBRS - genetic bleeding risk score, CBRS - clinical bleeding risk score

Beta $(B)$ is the multivariate coefficient value that reflects the relative weight of each factor in the final model. Exponential (Exp) Beta is the odds ratio or likelihood ratio of bleeding for each predictor in the model. Colinearity was checked and did not exist.

Table 2: Best fitting model in two cohorts using forward stepwise method of multivariate logistic regression.

\begin{tabular}{|c|c|c|c|}
\hline \multirow{2}{*}{ Predictor variables } & \multicolumn{3}{|c|}{ Scores assigned } \\
\hline & GBRS $^{A C}$ & GBRS $^{A C+W F}$ & CBRS $^{A C+W F}$ \\
\hline Factor V Leiden & - & 1 & - \\
\hline Age $>65$ years & 1 & 2 & 1 \\
\hline VKORC1 rs9934438 & 1 & 1 & - \\
\hline VKORC1 rs55894764 & 2 & - & - \\
\hline CYP2C9 rs1057911 & - & 1 & - \\
\hline Antiplatelet & - & - & 1 \\
\hline Cancer / malignancy $\$$ & 1 & 1 & 1 \\
\hline History of bleeding $\$$ & 1 & 1 & 1 \\
\hline Hepatic or renal disease ${ }^{\$}$ & 1 & 1 & 1 \\
\hline
\end{tabular}

\$Variables added in bleeding risk indices based on literature review of known bleeding risk factors in other indices such as HAS-BLED [14], HEMORR2HAGES [28], Shireman et al [27] and Kuijer et al [26]. However, these already established risk factors were not analyzed in the current population due to the study criteria.

Table 3: Scores assigned to the bleeding predictor variables in the three bleeding risk indices. 
and pooled cohort ${ }^{\mathrm{AC}+\mathrm{WF}}\left(\mathrm{GBRS}^{\mathrm{AC}+\mathrm{WF}}, \mathrm{CBRS}^{\mathrm{AC}+\mathrm{WF}}\right)$ are presented in Table 2. As the multivariate regression coefficient (B) value reflects the relative weight of each factor in the final model (Table 2), the scores allocated were based on these values (Table 3). Three important known risk factors of anticoagulant-induced bleeding (cancer/malignancy, history of bleeding and hepatic or renal disease) were added in the final bleeding risk indices based on literature review of bleeding risk factors in other indices such as HAS-BLED [14], HEMORRHAGES [28], Shireman et al. [27] and Kuijer et al. [26]. The risk scores for these three factors were allocated based on the weightage given to them in previous bleeding risk scores. These factors could not be analyzed in the present cohort due to the study criteria. Formulation of risk stratification of score values was done by computing scores for all patients in the derivation cohorts and plotting ROC graphs to determine their discriminative powers. With the ascending order of risk (low to high), a consistent increase in the per cent of bleeders was recorded with all three prediction algorithms, however the CBRS showed least percentage of actual bleeding among the patients with high bleeding risk score (Table 4). The bleeding risk prediction scores resulted in significant AUCs with both GBRSs (Table 5). As expected, the GBRS ${ }^{A C}$ showed enhanced AUC values in Cohort ${ }^{\mathrm{AC}}$ while GBRS ${ }^{\mathrm{AC}+W F}$ showed superior AUC plots in its derivation cohort ${ }^{\mathrm{AC}+\mathrm{WF}}$ as compared to that in other cohorts. Of the two genetic algorithms, GBRS ${ }^{\mathrm{AC}+\mathrm{WF}}$ was observed to be more accurate in its bleeding prediction in patients on warfarin, patients with DVT, and patients who were on concomitant antiplatelet therapy. Both GBR scores performed similarly in the cohort ${ }^{\mathrm{AC}}$, patients with clinical indications other than DVT and patients on OAC without concomitant antiplatelet therapy.

\section{Validation}

In the validation cohort, 13 (25.5\%) patients experienced bleeding (Supplementary Table 2). The high and low risk groups determined by the three prediction algorithms in the validation cohort (Table 6) revealed that $\mathrm{GBRS}^{\mathrm{AC}+\mathrm{WF}}$ demonstrated the lowest baseline risk of bleeding among the low risk group (17.8\%) and correctly identified highest proportion of bleeders $(83.3 \%)$ among the high risk groups. The GBRS ${ }^{\mathrm{AC}+\mathrm{WF}}$ proved to be the best prediction algorithm for bleeding as it had the highest sensitivity (38.5\%), positive predictive value (83.3), negative predictive rate (NPR; $82.2 \%$ ) with lowest false positive rate (FPR; 16.7\%) and false negative rate (FNR; $17.8 \%$ ) as compared to the other two indices. The clinical bleeding risk score (CBRS) performed poorly with the lowest sensitivity (15.4\%) and PPV (40.0\%) with highest FPR $(60 \%)$. Due to the limited number of clinical factors in the CBRS, it's AUC $(0.611$; CI 0.438-0.585) was much lower than those observed with genetic algorithms; GBRS ${ }^{A C}(0.719$; CI 0.544-0.894) and GBRS $^{\mathrm{AC}+\mathrm{WF}}(0.757 ; \mathrm{CI} 0.600-0.914)$.

\section{Discussion}

The study adds new knowledge with regards to important genetic and non-genetic predictors of bleeding risk. Integration of these bleeding predictors in the routine anticoagulation management could help caution the clinician against prescription of high doses in the high risk patients. The high risk patient group may also benefit from close and frequent monitoring of INR, lower intensity of anticoagulation (low target INR) or an alternate new oral anticoagulant. These measures can effectively reduce the number of bleeding episodes, thus sparing the patients from adverse outcomes and reducing the economic burden of hospital admissions due to anticoagulant related ADRs. The present study is the first to devise and validate a genetic scoring scheme for predicting bleeding among first time users of oral anticoagulants. The GBRS uses only some variables that are easily obtained from new patients (age, history of malignancy/cancer, bleeding, hepatic or renal disease), along with two or three genetic markers (GBRS ${ }^{\mathrm{AC}}$ and GBRS ${ }^{\mathrm{AC}+\mathrm{WF}}$ respectively). The addition of genetic variables was observed to increase the prediction sensitivity by twofolds as compared to use of clinical and demographic variables alone (Table 6). Although, the sample size of warfarin users was small $(n=53)$ and a separate algorithm could not be derived, the GBRS ${ }^{\mathrm{AC}+\mathrm{WF}}$ derived from the pooled Cohort ${ }^{\mathrm{AC}+\mathrm{WF}}$ proved to effectively distinguish bleeders from non-bleeders among warfarin users (Table 5). The GBRS $^{\mathrm{AC}+\mathrm{WF}}$ was observed to be the best scoring scheme by all statistical measures (Table 6) as well as the preferred score for use in patients on either types of coumarin derivatives, patients with DVT as clinical indication and patients on concomitant antiplatelet therapy (Table 5). The GBRS ${ }^{A C+W F}$ also showed lower FNR and higher PPV when validated in an independent cohort on acenocoumarol therapy (Table 6) as compared to that observed in the derivation cohort (Table 4). The overall better performance of the GBRS ${ }^{\mathrm{AC}+W F}$ over $\mathrm{GBRS}^{\mathrm{AC}}$ may be due to the higher frequency of CYP2C9 rs1057911 variant allele (0.105) in the study population as compared to VKORC1 rs55894764

\begin{tabular}{|c|c|c|c|c|c|c|c|c|c|}
\hline \multirow{2}{*}{ Predicted risk } & \multicolumn{3}{|c|}{ GBRS $^{A C}$} & \multicolumn{3}{|c|}{ GBRS $^{A C+W F}$} & \multicolumn{3}{|c|}{ CBRS $^{A C+W F}$} \\
\hline & Score & $\mathbf{N}$ & Actual Bled (\%) & Score & $\mathbf{N}$ & Actual Bled (\%) & Score & $\mathbf{N}$ & Actual Bled (\%) \\
\hline Low & $0-1$ & 189 & 22.2 & $0-2$ & 240 & 24.2 & $0-1$ & 237 & 24.5 \\
\hline High & $>2$ & 17 & 76.5 & $>3$ & 19 & 78.9 & 2 & 22 & 68.2 \\
\hline
\end{tabular}

The GBRS ${ }^{A C}$ was derived from the patients on acenocoumarol $(N=206)$; while $G B R S^{A C+W F}$ and $C B R S^{A C+W F}$ were derived from the pooled cohort $(N=259)$.

Table 4: Bleeding risk stratification of the two genetic bleeding risk scores (GBRS) and Clinical Bleeding risk score (CBRS).

\begin{tabular}{|c|c|c|c|c|c|c|}
\hline \multirow{2}{*}{ Cohorts } & \multirow{2}{*}{$\mathbf{N}$} & \multirow{2}{*}{$\%$ bled } & \multicolumn{2}{|c|}{ GBRS $^{A C}$} & \multicolumn{2}{|c|}{ GBRS $^{A C+W F}$} \\
\hline & & & AUC & $(95 \% \mathrm{Cl})$ & AUC & $(95 \% \mathrm{Cl})$ \\
\hline Either oral anticoagulant (OAC) & 259 & $73(28.2)$ & 0.640 & $(0.561-0.719)$ & 0.706 & $(0.633-0.778)$ \\
\hline Acenocoumarol & 206 & $55(26.7)$ & 0.671 & $(0.583-0.760)$ & 0.666 & $(0.579-0.753)$ \\
\hline Warfarin & 53 & $18(33.9)$ & 0.567 & $(0.400-0.735)$ & 0.855 & $(0.742-0.967)$ \\
\hline Deep vein thrombosis & 132 & $41(31.1)$ & 0.676 & $(0.570-0.781)$ & 0.802 & $(0.722-0.882)$ \\
\hline Other clinical indications & 127 & $32(25.2)$ & 0.607 & $(0.487-0.726)$ & 0.591 & $(0.470-0.713)$ \\
\hline OAC + Antiplatelet & 157 & $53(33.8)$ & 0.618 & $(0.521-0.715)$ & 0.711 & $(0.625-0.797)$ \\
\hline OAC - Antiplatelet & 102 & $20(19.6)$ & 0.692 & $(0.556-0.828)$ & 0.657 & $(0.511-0.803)$ \\
\hline
\end{tabular}

GBRS - genetic bleeding risk score; Area under the curve (AUC) denotes the proportion of cases that were accurately distinguished into the correct risk categories of bleeding. The AUC in bold font showed significantly higher values with GBRS ${ }^{A C+W F}$. OAC (Oral anticoagulant)

Table 5: A comparison of the discriminative power of the two logistic equations calculated as the area under curve (AUC) in different groups within the cohort 
variant allele (0.010) (Table 2) included in the GBRS ${ }^{\mathrm{AC}+\mathrm{WF}}$ and GBRS ${ }^{\mathrm{AC}}$ respectively. Although the sensitivities of both GBRSs are modest, the high specificity (Table 6) suggests that the scores are able to correctly identify patients who are not likely to bleed. This low sensitivity could be due to the unequal proportions of cases and controls in the study (27.7\% cases and rest served as controls).

Older age ( $>60 />65$ years) has been consistently associated with increased bleeding risk in literature and therefore included in most bleeding risk stratification schemes [13,26-28] as well as the present scoring scheme. Concomitant use of antiplatelet drugs, that was identified as a significant risk factor for bleeding (in univariate analyses) in the present study has also been identified frequently in previous studies $[27,50,51]$. There are mixed reports of the role of comorbidities in anticoagulation-related bleeding. Although hypertension, diabetes [51], and stroke $[13,14]$ have been included in some other bleeding risk scores, a large systematic literature review of 41 studies that evaluated the association of diabetes, hypertension, older age, chronic heart failure and cerebrovascular disease with anticoagulation related bleeding [52] observed low strength of evidence for all these factors except older age which showed moderate strength of association. A similar trend is reflected in the present study as well. Our findings did not show increased bleeding in females as has been reported previously $[26,27,51]$. Few other factors that have been correlated with bleeding in literature, such as history of stroke $[13,14]$ and smoking [51] failed to replicate a similar relationship in our cohort. The higher number of INR measurements and longer time taken to stabilize among bleeders implies that those who bled required more frequent INR monitoring (possibly due to fluctuating INRs) as compared to controls. This is parallel to the direct correlation of the proportion of out-of-range INRs $(>3.0,<2.0)$ and the risk of bleeding. Although 'labile INRs' (therapeutic time in range $<60 \%$ ) is one of the bleeding predictors in the HAS-BLED score index [14], we did not analyse it in the present study as the GBR score was primarily aimed for coumarin-naive patients.

CYP2C9 variants have been previously associated with 'major' or 'severe and life threatening' bleeding events (but not with minor bleeding) in patients on acenocoumarol [53] and warfarin [10,12,54]. The present study showed no significant increase in the likelihood of bleeding among the ${ }^{\star} 2$ or ${ }^{\star} 3$ carriers, akin to a few other studies with warfarin $[34,55]$. This may be due to inclusion of all types of bleeding (minor and major) in the derivation of GBRS. Also, the frequency of CYP2C $9{ }^{\star} 2$ and ${ }^{*} 3$ variants in the Asian-Indian population is reported to vary from other ethnic populations [56]. However, a significant association of ${ }^{*} 3$ allele was observed specifically with major bleeding $(n=18)$. But, due to the small numbers of major bleeding events, the validity of this association is questionable in the present population. A synonymous variant (rs1057911) reported to be in strong linkage disequilibrium with $* 3$ [57], and also classified as a tag SNP to identify $C Y P 2 C 9^{\star} 18$ haplotype (rs1057910, rs1057911, rs72558193) was observed to show highly significant marker for bleeding in the present study. This polymorphism was reported to be significantly associated with warfarin dose variance, explaining $14.5 \%$ of variance [58]. Although the exact mechanism of the role of this polymorphism in bleeding is unknown, synonymous changes are being increasingly reported and are known to affect the translation efficiency by interfering with both mRNA stability and the protein translation rate $[59,60]$. Two intronic CYP2C9 polymorphisms- rs9332230 and rs9332172 showed high prevalence in the cases as compared to controls. There are no previous reports of similar association. The mechanism of this effect is unknown, however it is likely that these variants could be linked to other non-synonymous SNPs (such as CYP2C $9 * 3$ and ${ }^{*}$ ) or modify the mRNA expression, transcription or regulation of the CYP2C9 enzyme that may result in slower drug metabolism and enhanced pharmacologic activity leading to toxicity (bleeding). Further in vitro functional and pharmacokinetic studies are warranted to confirm their role in anticoagulant induced bleeding.

Polymorphisms in VKORC1; rs9934438 $(1173 \mathrm{C}>\mathrm{T})$ and rs55894764 were significantly associated with acenocoumarolinduced bleeding but not with warfarin. However, $1173 \mathrm{C}>\mathrm{T}$ withstood multivariate regression analyses and showed significant predictability in both genetic algorithms (GBRS $\left.{ }^{A C}, G B R S^{A C+W F}\right)$. Despite the small sample size of warfarin users in the current study, the absence of correlation of $V K O R C 1$ variants with warfarin-induced bleeding has been reported previously as well [12]. A high haemorrhagic risk (but not statistically significant) has been reported with $1173 \mathrm{C} / \mathrm{T}$ variant in warfarin-users [12]. Another study [11] observed increased bleeding in Phenprocoumon users who carried the $1173 \mathrm{~T}$ allele. Thus, the present study is the first report of significant association of VKORC1 1173C $>\mathrm{T}$ with acenocoumarol-induced bleeding.

Factor V Leiden mutation, a known genetic risk factor for thrombophilia [38] was unexpectedly observed to increase bleeding risk among patients on anticoagulant therapy in the current study. Similar observation was made by Castori et al. [39]. Although, the exact mechanism by which FVL could cause bleeding is not known, some previous observations seem to support its prohemorrhagic role. A high frequency of the FVL mutation was reported in cases of hemorrhagerelated preterm delivery [61] and intraventricular hemorrhage in preterm infants $[62,63]$. The relatively hypercoaguable state in normal pregnancy and the protein $\mathrm{C}$ and $\mathrm{S}$ deficiency among the preterm infants is analogous to the state of anticoagulated patients taking coumarin derivatives. It is hypothesized that FVL thrombophilic mutation may aggravate this hemostasis shift and heighten the risk of clots in such patients and may someway facilitate the rupture of delicate blood vessels, resulting in hemorrhage [61]. Another (less likely) explanation could be linkage disequilibrium of the FVL mutation with unknown genetic variants that can alter the bleeding propensity while on oral anticoagulants [62-64]. Previous studies have shown that about $9.7 \%$ of FVL carriers may also have a combination of two or more variant

\begin{tabular}{|c|c|c|c|c|c|c|c|c|c|}
\hline Algorithm & Predicted risk & Bleeding N (\%) & No bleeding $\mathbf{N}(\%)$ & Sensitivity & Specificity & FPR & FNR & PPV & NPV \\
\hline \multirow{2}{*}{ GBRS $^{A C}$} & Low & $9(19.6)$ & $37(80.4)$ & \multirow{2}{*}{30.8} & \multirow{2}{*}{97.4} & \multirow{2}{*}{20.0} & \multirow{2}{*}{19.6} & \multirow{2}{*}{80.0} & \multirow{2}{*}{80.4} \\
\hline & High & $4(80.0)$ & $1(20.0)$ & & & & & & \\
\hline \multirow{2}{*}{$\mathrm{GBRS}^{\mathrm{AC}+\mathrm{WF}}$} & Low & $8(17.8)$ & $37(82.2)$ & \multirow{2}{*}{38.5} & \multirow{2}{*}{97.4} & \multirow{2}{*}{16.7} & \multirow{2}{*}{17.8} & \multirow{2}{*}{83.3} & \multirow{2}{*}{82.2} \\
\hline & High & $5(83.3)$ & $1(16.7)$ & & & & & & \\
\hline \multirow{2}{*}{$\mathrm{CBRS}^{\mathrm{AC}+\mathrm{WF}}$} & Low & $11(23.9)$ & $35(76.1)$ & \multirow{2}{*}{15.4} & \multirow{2}{*}{92.1} & \multirow{2}{*}{60.0} & \multirow{2}{*}{23.9} & \multirow{2}{*}{40.0} & \multirow{2}{*}{76.1} \\
\hline & High & $2(40.0)$ & $3(60.0)$ & & & & & & \\
\hline
\end{tabular}

GBRS - genetic bleeding risk score, CBRS - clinical bleeding risk score, FPR -false positive rate, FNR -false negative rate, PPV-positive predictive value, NPV -negative predictive value, AUC -area under the curve in receiver operator plot.

Table 6: Validation of the bleeding prediction models in an independent cohort on acenocoumarol therapy. 
CYP2C9/VKORC1 alleles [65], rendering them at very high risk of anticoagulation-induced bleeding.

The ABCB1 2677 TT and GT haplotypes previously associated with higher acenocoumarol dose [35] was observed to have a protective effect against bleeding complications in the current study. Although this is the first report documenting the protective effect of $A B C B 12677$ variant, a previous study [34] documents lesser prevalence of $A B C B 1$ variants among patients with warfarin-induced bleeding.

Pharmacokinetic drug interactions leading to bleeding with warfarin or acenocoumarol could occur with drugs that inhibit CYP2C9 or CYP2C19, affecting the drug concentrations, and subsequently enhancing its pharmacologic effect. From the pharmacodynamics perspective, drug interactions could also occur with drugs that interfere with platelet aggregation or synthesis of clotting factors, resulting in a synergistic effect. In the present study, all cases where concomitant drugs were observed to increase the likelihood of bleeding, respective patient records were analysed comprehensively for confirming the hemorrhagic role of drug-drug interactions. Use of Naranjo's probability scale [66] indicated 'probable' (scores 5-8) or 'possible' (14) relationships of the respective drug in combination with coumarin in all suspected cases of drug-drug-interaction.

Co-administration of proton pump inhibitors (PPIs, Pantoprazole and Rabeprazole) has occasionally been associated with potentiation of acenocoumarol $[67,68]$ and less commonly with warfarin [69-71]. However, until now bleeding was not analyzed as an outcome with co-administration of PPI in OAC users. The current study suggests a moderately significant role of interaction of PPIs with acenocoumarol but not with warfarin. As PPIs are essentially metabolized by CYP2C19 (and sometimes CYP2C9 and CYP3A4) which is partially involved in the metabolic clearance of the potent R-acenocoumarol [72], the resulting inhibition of CYP2C19 may reduce the clearance of acenocoumarol [73], leading to toxicity as observed in current study. On the other hand, the potent S-enantiomer of warfarin is metabolised by CYP2C9, which is inhibited to a lesser degree than CYP2C19.

Concomitant use of statin with OAC is reported to increase the anticoagulant response ensuing elevated INRs, dose reductions (10\%$27 \%$ ) and/or toxicity [74-76]. This is known to be caused by inhibition of CYP3A4 enzyme by statin and displacement of coumarin drug from protein binding site. On the other hand, a large study on warfarin users with atrial fibrillation has reported that long-term statin use ( $>1$ year) may be associated with a decreased risk of bleeding [77]. Our findings reiterate the protective effect of statin against bleeding complications among patients on long term warfarin use. However, this significant effect was not observed with acenocoumarol. It is likely that different generic drugs vary the role of statin interaction in bleeding with anticoagulants. Nonetheless, the small sample size of warfarin cohort limits the validity of the finding and will need to be replicated in a larger cohort on warfarin therapy before any conclusions or extrapolations can be drawn from the finding.

\section{Limitations}

a. The present study is limited in not analysing some already established bleeding risk factors such as alcohol abuse [14,27,28] due to insufficient data. Also, history of previous bleeding $[13,14,27,28]$, liver and renal disease [14,28] were not analyzed in the present cohort as they were a part of the exclusion criteria to enable us to focus the study on exploring the genetic predictors of bleeding. Exclusion of the above recognized non-genetic risk factors from the study may make it difficult to judge the true relative effect of genetic factors in a clinical population. Therefore, we present a more extensive bleeding risk stratification scheme that incorporates the above factors along with the novel genetic risk factors reported in the present study (Table 3). Three important known risk factors of anticoagulantinduced bleeding (cancer/malignancy, history of bleeding and hepatic or renal disease) were added in the final bleeding risk indices based on literature review of bleeding risk factors in other indices. Although these additional factors could not be analyzed in the current population, several studies have reported their important contribution to anticoagulant-induced bleeding in patients. Thus, this enhances the predictive power and scope of the current GBRS.

b. The study assumed a drug class effect for statins, antiplatelets and PPIs because of the exploratory nature of our study and the small frequency of patients on each type of generic drug. Preparationspecific protective or adverse effects could occur. For example, literary evidence of interaction of different types of statins with warfarin indicates that the warfarin interaction potential is more pronounced for simvastatin [74-76] than for atorvastatin or other statins [78]. Statins were observed to be one of the most commonly used drugs in the current study ( $\mathrm{n}=62,23.9 \%)$, however due to the limited number of patients on therapy with different generic forms of statin, we could not analyze their independent effect on bleeding outcome. Therefore, we cannot exclude the possibility that the effects we observed with statins may differ according to individual statin preparations.

c. Small sample size of patients on warfarin as compared to acenocoumarol limits the strength of bleeding risk factors that were observed exclusively in the cohort ${ }^{\mathrm{WF}}$. Larger studies analyzing bleeding complications with warfarin will be required to confirm some of the current findings.

d. Some dissimilarity in bleeding predictors was observed with the type of OAC i.e. warfarin and acenocoumarol (Table 1). Additionally, the quality of anticoagulation with the long-acting warfarin and the rapid-acting acenocoumarol differed in some aspects. The mean proportion of INR within therapeutic range was significantly greater among patients on warfarin than those on acenocoumarol (Supplemental Table 4). Occurrence of bleeding events appears to be higher in warfarin users but the difference was not statistically significant. This may be due to the longer mean follow up in the warfarin cohort. Also, the per cent of non-therapeutic INR $(<2.0)$ was lower with warfarin than acenocoumarol with borderline significance. A comparative study of quality and hemorrhagic risk with warfarin and acenocoumarol revealed that patients treated with acenocoumarol had a higher risk of presenting with an INR $\geq 6$, however no statistically significant differences were reported in therapeutic stability [79]. At present we have no clear explanation for risk differences between the two coumarin anticoagulants. More likely, the difference in bleeding predictors may be explained by the diverse pharmacokinetics of acenocoumarol and warfarin. The two coumarin derivatives have variable maintenance dose (lower for acenocoumarol), plasma concentration (lower with acenocoumarol), plasma clearance (faster with acenocoumarol), terminal elimination half life (shorter with acenocoumarol) and elimination kinetics (biphasic for acenocoumarol) [80]. 
Most importantly, the pharmacogenetic variability among the coumarins is likely to cause differential protein-drug binding and different drug-drug interactions that may in turn attribute to variation in genetic bleeding predictors with the two coumarin anticoagulants.

\section{Conclusions}

Genetically determined pharmacokinetic and pharmacodynamic capacity in an individual can dramatically alter the toxin and metabolite levels from those normally expected, which is crucial for drugs with a narrow therapeutic index, like acenocoumarol and warfarin. Genetic screening for bleeding predictors using simple scoring method have the potential to remove some of the scientific uncertainties in toxicity cases and can greatly reduce the economic burden of adverse drug reactions. However, the cost versus benefit of introducing such a form of genetic prediction will need to be further studied depending on the population incidence of bleeding and the cost of the rapid genetic test. It has been reported that a $6.9 \%$ improvement in the time spent within therapeutic range significantly reduced major hemorrhage by one event per 100 patient-years of treatment [81]. Hence, predictive bleeding scoring index along with improvement in the quality of anticoagulation by careful INR monitoring, proper management guidelines and patient education regarding concomitant drugs, vitamin $\mathrm{K}$ diet and signs of bleeding can decrease the incidence of bleeding complications.

\section{Executive Summary}

- The incidence rate was $21.32,16.86$ and 4.46 per 100 personyears for any type of bleeding, minor bleeding and major bleeding respectively.

- Six bleeding predictors were identified in patients on long-term warfarin therapy (CYP2C9- rs1057911, rs9332230, rs9332172; F5 rs6025, antiplatelet and statin drugs).

- Five bleeding predictors were identified in patients on long-term acenocoumarol therapy (VKORC1- rs55894764, rs9934438; $A B C B 1$ rs2032582, proton pump inhibitors and age $\geq 65$ years).

- Genetic Bleeding Risk Score (GBRS) ${ }^{\mathrm{AC}+\mathrm{WF}}$ identified $78.9 \%$ of bleeders as the 'high risk' group and demonstrated an area under the curve (AUC) of 0.855 in patients on warfarin, 0.706 in patients on either oral anticoagulant and 0.802 in patients with deep vein thrombosis. GBRS ${ }^{\mathrm{AC}+\mathrm{WF}}$ had a specificity of $97.4 \%$, false positive rate of $16.7 \%$ and false negative rate of $17.8 \%$.

- The GBRS was validated to perform better than the Clinical (nongenetic) Bleeding Risk Score (CBRS). The sensitivity increased two-folds with GBRS ${ }^{\mathrm{AC}+\mathrm{WF}}$ as compared to CBRS.

\section{Conclusions}

- Genetic screening for bleeding risk using the current simple scoring method has the potential to remove some of the scientific uncertainties in toxicity cases.

- Predictive bleeding score along with improvement in the quality of anticoagulation by careful INR monitoring, proper management guidelines and patient education regarding concomitant drugs and signs of bleeding can decrease the incidence of bleeding events. This can greatly reduce the economic burden of adverse drug reactions.

\section{Financial Disclosure/Conflict of interest}

Ishwar C Verma, Renu Saxena and Risha Nahar had received research grant for the above work from the Sir Ganga Ram Hospital. For the remaining authors no conflicts of interest are declared.

\section{Acknowledgements}

The authors are thankful to the Sir Ganga Ram Hospital for funding. The funding source was not involved in the study design, analysis and interpretation of data or in the writing of the manuscript.

\section{References}

1. Hemker HC, Frank HL (1985) The mechanism of action of oral anticoagulants and its consequences for the practice of oral anticoagulation. Haemostasis 15 263-270.

2. Wardrop D, Keeling $D(2008)$ The story of the discovery of heparin and warfarin Br J Haematol 141: 757-763.

3. Patel KJ, Kedia MS, Bajpai D, Mehta SS, Kshirsagar NA, et al. (2007) Evaluation of the prevalence and economic burden of adverse drug reactions presenting to the medical emergency department of a tertiary referral centre: a prospective study. BMC Clin Pharmacol 7: 8.

4. Pattanaik S, Dhamija P, Malhotra S, Sharma N, Pandhi P (2009) Evaluation of cost of treatment of drug-related events in a tertiary care public sector hospital in Northern India: a prospective study. Br J Clin Pharmacol 67: 363-369.

5. Zaidenstein R, Eyal S, Efrati S, Akivison L, Michowitz MK, et al. (2002) Adverse drug events in hospitalized patients treated with cardiovascular drugs and anticoagulants. Pharmacoepidemiol Drug Saf 11: 235-238.

6. Pirmohamed M, James S, Meakin S, Green C, Scott AK, et al. (2004) Adverse drug reactions as cause of admission to hospital: prospective analysis of 18 820 patients. BMJ 329: 15-19.

7. Ogg MS, Brennan P, Meade T, Humphries SE (1999) CYP2C9*3 allelic variant and bleeding complications. Lancet 354: 1124.

8. Aithal GP, Day CP, Kesteven PJ, Daly AK (1999) Association of polymorphisms in the cytochrome P450 CYP2C9 with warfarin dose requirement and risk of bleeding complications. Lancet 353: 717-719.

9. Margaglione M, Colaizzo D, D’Andrea G, Brancaccio V, Ciampa A, et al. (2000) Genetic modulation of oral anticoagulation with warfarin. Thromb Haemost 84 775-778.

10. Higashi MK, Veenstra DL, Kondo LM, Wittkowsky AK, Srinouanprachanh SL, et al. (2002) Association between CYP2C9 genetic variants and anticoagulationrelated outcomes during warfarin therapy. JAMA 287: 1690-1698.

11. Reitsma PH, van der Heijden JF, Groot AP, Rosendaal FR, Büller HR (2005) A C1173T dimorphism in the VKORC1 gene determines coumarin sensitivity and bleeding risk. PLoS Med 2: e312.

12. Limdi NA, McGwin G, Goldstein JA, Beasley TM, Arnett DK, et al. (2008) Influence of CYP2C9 and VKORC1 1173C/T genotype on the risk of hemorrhagic complications in African-American and European-American patients on warfarin. Clin Pharmacol Ther 83: 312-321.

13. Beyth RJ, Quinn LM, Landefeld CS (1998) Prospective evaluation of an index for predicting the risk of major bleeding in outpatients treated with warfarin. Am J Med 105: 91-99.

14. Pisters R, Lane DA, Nieuwlaat R, de Vos CB, Crijns HJ, et al. (2010) A nove user-friendly score (HAS-BLED) to assess 1-year risk of major bleeding in patients with atrial fibrillation: the Euro Heart Survey. Chest 138: 1093-1100.

15. Sconce EA, Khan TI, Wynne HA, Avery P, Monkhouse L, et al. (2005) The impact of CYP2C9 and VKORC1 genetic polymorphism and patient characteristics upon warfarin dose requirements: proposal for a new dosing regimen. Blood 106: $2329-2333$

16. Gage BF, Eby C, Johnson JA, Deych E, Rieder MJ, et al. (2008) Use of pharmacogenetic and clinical factors to predict the therapeutic dose of warfarin Clin Pharmacol Ther 84: 326-331.

17. International Warfarin Pharmacogenetics Consortium, Klein TE, Altman RB Eriksson N, Gage BF, et al. (2009) Estimation of the warfarin dose with clinical and pharmacogenetic data. N Engl J Med 360: 753-764.

18. Roper N, Storer B, Bona R, Fang M (2010) Validation and comparison of pharmacogenetics-based warfarin dosing algorithms for application of pharmacogenetic testing. J Mol Diagn 12: 283-291.

19. van Schie RM, Wessels JA, le Cessie S (2011) Loading and maintenance dose 
Citation: Nahar R, Verma IC, Deb R, Saxena R, Takkar P, et al. (2013) Genetic Bleeding Risk Score (GBRS) for Patients on Oral Anticoagulant Therapy. Int J Genomic Med 1: 101. doi:10.4172/2332-0672.1000101

algorithms for phenprocoumon and acenocoumarol using patient characteristics and pharmacogenetic data. Eur Heart J. 32: 1909-191.

20. Borobia AM, Lubomirov R, Ramírez E, Lorenzo A, Campos A, et al. (2012) An acenocoumarol dosing algorithm using clinical and pharmacogenetic data in Spanish patients with thromboembolic disease. PLoS One 7: e41360.

21. Rathore SS, Agarwal SK, Pande S, Singh SK, Mittal T, et al. (2012) Therapeutic dosing of acenocoumarol: proposal of a population specific pharmacogenetic dosing algorithm and its validation in north Indians. PLoS One 7: e37844.

22. Kangelaris KN, Bent S, Nussbaum RL, Garcia DA, Tice JA (2009) Genetic testing before anticoagulation? A systematic review of pharmacogenetic dosing of warfarin. J Gen Intern Med 24: 656-664.

23. Hillman MA, Wilke RA, Yale SH, Vidaillet HJ, Caldwell MD, et al. (2005) A prospective, randomized pilot trial of model-based warfarin dose initiation using CYP2C9 genotype and clinical data. Clin Med Res 3: 137-145.

24. Caraco Y, Blotnick S, Muszkat M (2008) CYP2C9 genotype-guided warfarin prescribing enhances the efficacy and safety of anticoagulation: a prospective randomized controlled study. Clin Pharmacol Ther 83: 460-470.

25. Anderson JL, Horne BD, Stevens SM, Grove AS, Barton S, et al. (2007) Randomized trial of genotype-guided versus standard warfarin dosing in patients initiating oral anticoagulation. Circulation 116: 2563-2570.

26. Kuijer PM, Hutten BA, Prins MH, Büller HR (1999) Prediction of the risk of bleeding during anticoagulant treatment for venous thromboembolism. Arch Intern Med 159: 457-460.

27. Shireman TI, Mahnken JD, Howard PA, Kresowik TF, Hou Q, et al. (2006) Development of a contemporary bleeding risk model for elderly warfarin recipients. Chest 130: 1390-1396.

28. Gage BF, Yan Y, Milligan PE, Waterman AD, Culverhouse R, et al. (2006) Clinical classification schemes for predicting hemorrhage: results from the National Registry of Atrial Fibrillation (NRAF). Am Heart J 151: 713-719.

29. Corbo RM, Scacchi $\mathrm{R}$ (1999) Apolipoprotein $\mathrm{E}(A P O E)$ allele distribution in the world. Is $A P O E^{\star} 4$ a 'thrifty' allele? Ann Hum Genet 63: 301-310.

30. Kimmel SE, Christie J, Kealey C, Chen Z, Price M, et al. (2008) Apolipoprotein E genotype and warfarin dosing among Caucasians and African Americans. Pharmacogenomics J 8: 53-60.

31. Kohnke H, Sörlin K, Granath G, Wadelius M (2005) Warfarin dose related to apolipoprotein $\mathrm{E}(A P O E)$ genotype. Eur J Clin Pharmacol 61: 381-388.

32. Eichelbaum M, Fromm MF, Schwab M (2004) Clinical aspects of the MDR1 (ABCB1) gene polymorphism. Ther Drug Monit 26: 180-185

33. Pauli-Magnus C, Kroetz DL (2004) Functional implications of genetic polymorphisms in the multidrug resistance gene MDR1 (ABCB1). Pharm Res 21: $904-913$

34. Wadelius M, Sörlin K, Wallerman O, Karlsson J, Yue QY, et al. (2004) Warfarin sensitivity related to CYP2C9, CYP3A5, ABCB1 (MDR1) and other factors. Pharmacogenomics J 4: 40-48.

35. Saraeva RB, Paskaleva ID, Doncheva E, Eap CB, Ganev VS (2007) Pharmacogenetics of acenocoumarol: CYP2C9, CYP2C19, CYP1A2, $C Y P 3 A 4, C Y P 3 A 5$ and $A B C B 1$ gene polymorphisms and dose requirements. $J$ Clin Pharm Ther 32: 641-649.

36. Takeuchi F, McGinnis R, Bourgeois S, Barnes C, Eriksson N, et al. (2009) A genome-wide association study confirms VKORC1, CYP2C9, and CYP4F2 as principal genetic determinants of warfarin dose. PLoS Genet 5: e1000433.

37. Pérez-Andreu V, Roldán V, Antón Al, García-Barberá N, Corral J, et al (2009) Pharmacogenetic relevance of CYP4F2 V433M polymorphism on acenocoumarol therapy. Blood 113: 4977-4979.

38. Deitcher SR, Caiola E, Jaffer A (2000) Demystifying two common genetic predispositions to venous thrombosis. Cleve Clin J Med 67: 825-826, 829, 833-

39. Castori L, Taliani MR, Agnelli G, Boschetti E, Gresele P (2005) Interactions between thrombophilic genetic mutations and clinical bleeding in patients on chronic oral anticoagulant treatment. Haematologica 90: 1720-1722.

40. Veeger NJ, Piersma-Wichers M, Meijer K, Hillege HL (2011) Minor bleeds alert for subsequent major bleeding in patients using vitamin $\mathrm{K}$ antagonists. $\mathrm{Br}$ Haematol 153: 508-514.
41. Miller SA, Dykes DD, Polesky HF (1988) A simple salting out procedure for extracting DNA from human nucleated cells. Nucleic Acids Res 16: 1215.

42. D'Andrea G, D'Ambrosio RL, Di Perna P, Chetta M, Santacroce R, et al. (2005) A polymorphism in the VKORC1 gene is associated with an interindividual variability in the dose-anticoagulant effect of warfarin. Blood 105: 645-649.

43. Si D, Guo Y, Zhang Y, Yang L, Zhou H, et al. (2004) Identification of a novel variant CYP2C9 allele in Chinese. Pharmacogenetics. 14: 465-469.

44. You FM, Huo N, Gu YQ, Luo MC, Ma Y, et al. (2008) BatchPrimer3: a high throughput web application for PCR and sequencing primer design. BMC Bioinformatics 9: 253.

45. Hoffmeyer S, Burk O, von Richter O, Arnold HP, Brockmöller J, et al. (2000) Functional polymorphisms of the human multidrug-resistance gene: multiple sequence variations and correlation of one allele with $\mathrm{P}$-glycoprotein expression and activity in vivo. Proc Natl Acad Sci U S A 97: 3473-3478.

46. Kim RB, Leake BF, Choo EF, Dresser GK, Kubba SV, et al. (2001) Identification of functionally variant MDR1 alleles among European Americans and African Americans. Clin Pharmacol Ther 70: 189-199.

47. Ingelsson M, Shin Y, Irizarry MC, Hyman BT, Lilius L, et al. (2003) Genotyping of apolipoprotein E: comparative evaluation of different protocols. Curr Protoc Hum Genet Chapter 9: Unit9.

48. Sproul AM, Chalmers EA (2004) Multiplex PCR for the detection of the factor V Leiden and prothrombin 20210A mutations. Methods Mol Med 91: 79-87.

49. Poort SR, Rosendaal FR, Reitsma PH, Bertina RM (1996) A common genetic variation in the 3'-untranslated region of the prothrombin gene is associated with elevated plasma prothrombin levels and an increase in venous thrombosis. Blood 88: 3698-3703.

50. Shireman TI, Howard PA, Kresowik TF, Ellerbeck EF (2004) Combined anticoagulant-antiplatelet use and major bleeding events in elderly atria fibrillation patients. Stroke 35: 2362-2367.

51. Nekkanti $H$, Mateti UV, Vilakkathala R, Rajakannan T, Mallayasamy S, et al. (2012) Predictors of warfarin-induced bleeding in a South Indian cardiology unit. Perspect Clin Res 3: 22-25.

52. Chen WT, White CM, Phung OJ, Kluger J, Ashaye AO, et al. (2011) Association between CHADSâ,,risk factors and anticoagulation-related bleeding: a systematic literature review. Mayo Clin Proc 86: 509-521.

53. Visser LE, van Schaik RH, van Vliet M, Trienekens PH, De Smet PA, et al. (2004) The risk of bleeding complications in patients with cytochrome P450 CYP2C9*2 or CYP2C9*3 alleles on acenocoumarol or phenprocoumon. Thromb Haemost 92: 61-66.

54. Sanderson S, Emery J, Higgins J (2005) CYP2C9 gene variants, drug dose, and bleeding risk in warfarin-treated patients: a HuGEnet systematic review and meta-analysis. Genet Med 7: 97-104.

55. Taube J, Halsall D, Baglin T (2000) Influence of cytochrome P-450 CYP2C9 polymorphisms on warfarin sensitivity and risk of over-anticoagulation in patients on long-term treatment. Blood 96: 1816-1819.

56. Nahar R, Deb R, Saxena R, Puri RD, Verma IC (2013) Variability in CYP2C9 allele frequency: a pilot study of its predicted impact on warfarin response among healthy South and North Indians. Pharmacol Rep 65: 187-194.

57. Liao LH, Zhang H, Lai MP, Lau KW, Lai AK, et al. (2007) The association of CYP2C9 gene polymorphisms with colorectal carcinoma in Han Chinese. Clin Chim Acta 380: 191-196.

58. Wadelius M, Chen LY, Eriksson N, Bumpstead S, Ghori J, et al. (2007) Association of warfarin dose with genes involved in its action and metabolism. Hum Genet 121: 23-34

59. Kimchi-Sarfaty C, Oh JM, Kim IW, Sauna ZE, Calcagno AM, et al. (2007) A "silent" polymorphism in the MDR1 gene changes substrate specificity. Science 315: 525-528.

60. Duan J, Wainwright MS, Comeron JM, Saitou N, Sanders AR, et al. (2003) Synonymous mutations in the human dopamine receptor D2 (DRD2) affect mRNA stability and synthesis of the receptor. Hum Mol Genet 12: 205-216.

61. Gargano JW, Holzman CB, Senagore PK, Reuss ML, Pathak DR, et al. (2009) Polymorphisms in thrombophilia and renin-angiotensin system pathways preterm delivery, and evidence of placental hemorrhage. Am J Obstet Gyneco 201: 317. 
Citation: Nahar R, Verma IC, Deb R, Saxena R, Takkar P, et al. (2013) Genetic Bleeding Risk Score (GBRS) for Patients on Oral Anticoagulant Therapy. Int J Genomic Med 1: 101. doi:10.4172/2332-0672.1000101

62. Melegh B, Stankovics J, Kis A, Nagy A, Storcz J, et al. (1998) Increased prevalence of factor $\mathrm{V}$ Leiden mutation in neonatal intracranial haemorrhage. Eur J Pediatr 157: 261

63. Petäjä J, Hiltunen L, Fellman V (2001) Increased risk of intraventricular hemorrhage in preterm infants with thrombophilia. Pediatr Res 49: 643-646.

64. D’Ambrosio RL, D’Andrea G, Cappucci F, Chetta M, Di Perna P, et al. (2004) Polymorphisms in factor II and factor VII genes modulate oral anticoagulation with warfarin. Haematologica 89: 1510-1516.

65. Nahar R, Saxena R, Deb R, Verma IC (2012) Pharmacogenetic typing for ora anti-coagulant response among factor $\mathrm{V}$ Leiden mutation carriers. Indian $\mathrm{J}$ Hum Genet 18: 326-331.

66. Naranjo CA, Busto U, Sellers EM, Sandor P, Ruiz I, et al. (1981) A method for estimating the probability of adverse drug reactions. Clin Pharmacol Ther 30: 239-245

67. Garcia B, Lacambra C, Garrote FG-PI, Solis J (1994) Possible potentiaition of anticoagulant effect of acenocoumarol by omeprazole. Pharmacy World and Science. 16: 231-232.

68. Teichert M, van Noord C, Uitterlinden AG, Hofman A, Buhre PN, et al (2011) Proton pump inhibitors and the risk of overanticoagulation during acenocoumarol maintenance treatment. Br J Haematol 153: 379-385.

69. Duursema L, Müller FO, Schall R, Middle MV, Hundt HK, et al. (1995) Lack of effect of pantoprazole on the pharmacodynamics and pharmacokinetics of warfarin. Br J Clin Pharmacol 39: 700-703.

70. Wittkowsky AK (2001) Drug interactions update: drugs, herbs, and oral anticoagulation. J Thromb Thrombolysis 12: 67-71.

71. Holbrook AM, Pereira JA, Labiris R, McDonald H, Douketis JD, et al. (2005) Systematic overview of warfarin and its drug and food interactions. Arch Intern Med 165: 1095-1106
72. Meyer UA (1996) Interaction of proton pump inhibitors with cytochromes P450: consequences for drug interactions. Yale J Biol Med 69: 203-209.

73. Gschwind L, Rollason V, Lovis C, Boehlen F, Bonnabry P, et al. (2013) Identification and weighting of the most critical "real-life" drug-drug interactions with acenocoumarol in a tertiary care hospital. Eur J Clin Pharmacol 69: 617 627

74. Westergren T, Johansson P, Molden E (2007) Probable warfarin-simvastatin interaction. Ann Pharmacother 41: 1292-1295.

75. Hickmott H, Wynne H, Kamali F (2003) The effect of simvastatin co-medication on warfarin anticoagulation response and dose requirements. Thromb Haemost 89: 949-950.

76. Sconce EA, Khan TI, Daly AK, Wynne HA, Kamali F (2006) The impact of simvastatin on warfarin disposition and dose requirements. J Thromb Haemost 4. $1422-1424$

77. Douketis JD, Melo M, Bell CM, Mamdani MM (2007) Does statin therapy decrease the risk for bleeding in patients who are receiving warfarin? Am J Med 120: 369.

78. Stern R, Abel R, Gibson GL, Besserer J (1997) Atorvastatin does not alter the anticoagulant activity of warfarin. J Clin Pharmacol 37: 1062-1064.

79. Oliva Berini E, Galán Alvarez P, Pacheco Onrubia AM (2008) Comparison of quality and hemorragic risk of oral anticoagulant therapy using acenocoumaro versus warfarin. Med Clin (Barc) 131: 96-97.

80. Ufer M (2005) Comparative pharmacokinetics of vitamin $\mathrm{K}$ antagonists: warfarin, phenprocoumon and acenocoumarol. Clin Pharmacokinet 44: 12271246.

81. Wan Y, Heneghan C, Perera R, Roberts N, Hollowell J, et al. (2008) Anticoagulation control and prediction of adverse events in patients with atria fibrillation: a systematic review. Circ Cardiovasc Qual Outcomes 1: 84-91. 